\title{
Sellado de lesiones de caries dental no cavitadas: ensayo clínico aleatorio controlado
}

\section{Resumen}

Objetivo: Evaluar la efectividad del sellado de lesiones de caries dental no cavitadas. Materiales y Métodos: Se realizó un ensayo clínico, aleatorio, doble ciego, controlado y diseño de boca dividida. Fueron seleccionados 262 primeros molares permanentes, en 74 niños con edad media de 7,47 \pm 0.8 años. Cada paciente tenía mínimo un par de primeros molares permanentes con lesiones bilaterales de caries dental códigos 1, 2 o 3 del Sistema Internacional de Detección y Valoración de Caries Dental. Se asignaron aleatoriamente a tres tipos de tratamiento, 1: vidrio ionomérico (3M ESPE Ketac Molar Easymix®), 2: Sellante resinoso (3M ESPE Climpro $\left.{ }^{\circledR}\right)$, 3: vidrio ionomérico modificado con resina (3M ESPE Ketac ${ }^{\circledR}$ N100). La retención y progresión de caries dental fueron evaluadas. Los datos se procesaron con el programa SPSS V15. Se utilizaron las
Jesús Alberto-Luengo-Fereira ${ }^{1}$

Olga Raquel-Zambrano-Palencia ${ }^{2}$

Luis Eduardo-Rivera ${ }^{3}$ pruebas Chi Cuadrado y Test de Fisher. Resultados: La tasa retención total de los tratamientos 1 , 2 y 3 fue respectivamente: $51,7 \%, 43,4 \%$ y $26,4 \%$; no obstante estas diferencias no fueron significativas. La incidencia de caries en los molares no sellados fue $31,8 \%$ y en los molares sellados con perdida completa del material $28,5 \%$. No se encontraron diferencias significativas en la progresión de las lesiones entre molares que reciben y los que no reciben tratamiento. Conclusiones: En este estudio, no se encontraron evidencias de diferencias en la retención y en la progresión de las lesiones selladas y no selladas, entre el vidrio ionomérico convencional de alta densidad, el sellante a base de resina y un vidrio ionomérico modificado con resina.

Palabras clave: Caries Dental, lesión no cavitada, sellante, mínima intervención.

\section{Artigo Original}

\section{Selamento de lesões de cariosas não cavitadas: ensaio clínico controlado randomizado}

\section{Resumo}

Objetivo: Avaliar a efetividade do selamento de lesões cariosas não cavitadas. Materiais e Mé- todos: Realizou-se um ensaio clínico, randomizado, duplo cego, controlado e desenho experimental tipo boca dividida. Foram selecionados 262 primeiros molares permanentes, em 74 me-

\footnotetext{
${ }^{1}$ Magíster Scientiarum en Odontopediatría. Especialidad en Odontopediatría. Universidad Autónoma de Zacatecas, México. jluengofereira@gmail.com

${ }^{2}$ Doctora en Odontología. Instituto de Investigaciones de Odontología. Universidad del Zulia, Venezuela.ozambrano001@gmail.com

${ }^{3}$ Doctor en Odontología. Instituto de Investigaciones de Odontología. Universidad del Zulia, Venezuela.luiggi3175@gmail.com
} 
ninos com idade média de 7,47 \pm 0.8 anos. Cada paciente tinha mínimo dois primeiros molares permanentes com lesões bilaterais de caries dental códigos 1, 2 ou 3 do Sistema Internacional de Detecção e Avaliação de Caries Dentária. Atribuíram-se aleatoriamente três tipos de tratamento, 1: ionômero de vidro (3M ESPE Ketac Molar Easymix®), 2: Selante resinoso (3M ESPE Climpro®), 3: ionômero de vidro modificado com resina (3M ESPE Ketac® N100). A retenção e a progressão das lesões de cárie dentária foram avaliadas. Os dados foram analisados através do programa SPSS V15. Utilizaram-se as provas Chi Quadrado e Teste de Fisher. Resultados: A taxa de retenção total dos tratamentos 1, 2 e 3 foi respectivamente: $51,7 \%, 43,4 \%$ e $26,4 \%$; no en- tanto, estas diferenças não foram significativas. A incidência de cárie em os molares não selados foi $31,8 \%$ e em molares selados com perdida completa do material foi de $28,5 \%$. Não se encontraram diferenças significativas na progressão das lesões entre os molares que receberam e os que não receberam tratamento. Conclusões: Neste estudo, não se encontraram evidências de diferenças na retenção e na progressão das lesões seladas e não seladas, entre o ionômero de vidro convencional de alta densidade, o selante a base de resina e o ionômero de vidro modificado com resina.

Palavras-chave: Carie dentária, lesão não cavitada, selante, mínima intervenção.

\section{Original article}

\section{Sealing of non-cavitated dental caries lesions: random controlled clinical trial}

\begin{abstract}
Objective: To evaluate the effectiveness of sealing caries lesions non-cavitated. Materials and Methods: A clinical, randomized, double-blind, controlled, split-mouth design was performed. We selected 262 first permanent molars in 74 children with a mean age of $7.47 \pm 0.8$ years. Each patient had at least one pair of first permanent molars with bilateral lesions of codes 1 , 2 or 3 dental caries International Detection and Rating of Dental Caries. Were randomly assigned to three treatment types, 1: glass ionomer (3M ESPE Ketac Molar Easymix®), 2: resinous sealant (3M ESPE Climpro®), 3: resin-modified glass ionomer (3M ESPE Ketac ${ }^{\circledR}$ N100). Retention and progression of dental caries were evaluated. Data were processed with SPSS V15 pro-
\end{abstract}

gram. Chi Square test and Fisher test were used. Results: The overall retention rate treatments 1, 2 and 3 was respectively $51.7 \%, 43.4 \%$ and $26.4 \%$, but these differences were not significant. The incidence of caries in the unsealed molars was $31.8 \%$ and in the sealed molars with complete loss of the material $28.5 \%$. No significant differences in lesion progression between molars receiving and those not receiving treatment were found. Conclusions: In this study, no evidence of differences were found in the retention and progression of sealed and unsealed injuries, conventional glass ionomer high density, based sealant resin and a modified glass ionomer re$\sin$.

Key words: Dental caries, non-cavitated lesion, minimal intervention, sealant. 


\section{Introducción}

El proceso de la caries ocurre por la interacción de la placa dental y la superficie del diente a través del tiempo, donde las bacterias que se encuentran en la bio-película son metabólicamente activas, causando fluctuaciones en el $\mathrm{pH}$ del fluido de la placa, originando pérdida de minerales del diente cuando los niveles de $\mathrm{pH}$ disminuyen; o una ganancia de los mismos, cuando éstos niveles se incrementan ${ }^{1}$.

La caries puede controlarse desde sus etapas iniciales, sin embargo, a pesar de la existencia e implementación de diferentes métodos para lograr éste cometido, sigue siendo una de las enfermedades de mayor prevalencia en la población mundial, especialmente en la infantil ${ }^{2}$. Las lesiones aparecen principalmente en las fosas y fisuras oclusales de molares permanentes en un $90 \%^{3}$, debido a su compleja morfología; convirtiéndolas en zonas de difícil acceso para la auto-limpieza o el cepillado dental; y de mayor susceptibilidad al acumulo de placa, produciendo así, que el esmalte no reciba los mismos niveles de fluoruro y de antimicrobianos, en comparación con las superficies lisas ${ }^{4}$. De igual manera, la acumulación de placa y susceptibilidad a la caries son mayores durante la erupción de los molares, aunado a la susceptibilidad de cada individuo, hacen que estas superficies sean vulnerables para el inicio temprano y la rápida progresión de la caries ${ }^{5}$.

Una de las estrategias para prevenir la aparición y controlar la progresión de las lesiones cariosas en las superficies oclusales, es el uso de selladores de fosas y fisuras ${ }^{6}$. Estos materiales previenen la colonización de bacterias cariogénicas, al formar una capa protectora de adhesión micromecánica a la superficie del esmalte, suprimiendo el acceso de los microorganismos a las zonas susceptibles ${ }^{1}$.
Actualmente, se conocen dos tipos de materiales empleados como selladores: de resina (son los más utilizados y los de primera elección) y de ionómero de vidrio (son una alternativa en aquellos casos donde el control de la humedad se encuentra limitado o es difícil de lograr ${ }^{1,5}$, además, liberan iones de fluoruro al esmalte adyacente y absorben fluoruro de otras fuentes, como dentífricos y enjuagues bucales, comportándose en la cavidad oral, como dispositivos de recarga y de liberación progresiva de fluoruro ${ }^{7}$.

A pesar de lo expuesto, revisiones sistemáticas de Ahovuo-Saloranta y col. ${ }^{8}$ y Azarpazhooh y col. ${ }^{9}$, indican que la efectividad de la aplicación de los sellantes de fosas y fisuras, para prevenir caries dental en los dientes posteriores de niños entre 6 y 17 años de edad con alto riesgo a desarrollar la enfermedad, se encuentra relacionada con la retención del material a largo plazo en su sitio de aplicación; ya que, cuando el sellador se pierde o la fisura que estaba sellada queda parcialmente descubierta, los fluidos orales pueden migrar a la fisura y/o debajo del sellador, y así posiblemente interactuar con las bacterias de la placa dental o con las de una lesión cariosa inactiva, produciendo que el proceso carioso pase de nuevo a un estado activo ${ }^{10}$.

Oong y $\mathrm{CO}^{11}$, en una revisión de la literatura sobre el efecto de los selladores en los niveles de bacterias de lesiones de caries dental, reportan que las aplicaciones de selladores son capaces de disminuir hasta en un $50 \%$ los niveles de bacterias reduciendo la progresión de la enfermedad. Beauchamp y col. ${ }^{1}$, indican que la disminución en la incidencia de caries dental después de la colocación de un sellador va desde un $86 \%$ al año, $78.6 \%$ a los 2 años y $56.6 \%$ a los 4 años. Un metanálisis realizado por Griffin y col. ${ }^{12}$, sobre la efectividad de los sellantes en la prevención del progreso de lesiones de caries no cavitadas, su- 
gieren que el sellado de estas lesiones es efectivo para reducir su avance, mostrando una media anual de progresión de $2.6 \%$ en los molares sellados; $12.6 \%$ en molares no sellados. Mostrando una fracción de prevención de 71.3\% luego de 5 años.

Sin embargo, el efecto clínico sobre la liberación de fluoruro de los cementos de vidrio ionomérico no está bien establecido. Los estudios clínicos han proporcionado pruebas contradictorias acerca de si estos materiales pueden prevenir o inhibir significativamente la caries, o si afectan el crecimiento de las bacterias asociadas a la caries, en comparación con los materiales que no contienen fluoruro ${ }^{1}$. No obstante, existen estudios que muestran una mayor efectividad de los selladores de vidrio ionomérico sobre los de resina, tanto en su técnica de aplicación como para evitar el progreso de lesiones cariosas no cavitadas.

Salar y col. ${ }^{13}$, en un ensayo clínico, aleatorio, compararon tres tipos de sellantes: uno resinoso, sellante resinoso que libera fluoruro y otro de vidrio ionomérico. Este estudio demostró que los sellantes resinosos que liberaban fluoruros inhiben la desmineralización del esmalte adyacente al material, pero menos que aquellos de vidrio ionomérico. Trairatvorakul y col. ${ }^{14}$, reportan que los selladores de ionómero de vidrio son capaces de reducir la incidencia de lesiones cariosas $0.5 \mathrm{~mm}$ en las adyacencias de los materiales. Kantovitz y col. ${ }^{15}$, concluyen que los selladores resinosos no previenen la desmineralización del esmalte, contrario a los de vidrio ionomérico, los cuales muestran una mayor capacidad de liberación de fluoruros.

Otro estudio realizado por Beiruti y col. ${ }^{16}$, sobre el efecto preventivo de los sellantes, indica que luego de 5 años de evaluación, el $88 \%$ y el $86 \%$ de los sellantes de resina y los de vidrio ionó- mero (aplicados con la técnica ART), se habían perdido respectivamente. Así mismo, posterior a los 2 y 3 años de la pérdida de material, las lesiones de caries de dentina aparecieron en un $13 \%$ en el grupo de sellantes de resina y $3 \%$ en el grupo de sellantes de vidrio ionomérico. Los autores concluyeron, en que el sellante de vidrio ionomérico parece tener 4 veces más oportunidad de prevenir el desarrollo de caries en fosas y fisuras que los sellantes resinosos.

Beiruti y col. ${ }^{17}$ en una revisión sistemática, sobre el efecto preventivo de los sellantes a base de resina y de vidrio ionomérico para prevenir el desarrollo de lesiones de caries en el esmalte de dientes primarios y permanentes, indica que no existe evidencia suficiente sobre que material es superior al otro, si se toma en cuenta el riesgo de la población a presentar caries de fosas y fisuras.

Komatsu y col. ${ }^{18}$, señalan que la reaplicación de los selladores de vidrio ionomérico favorecen la reducción de lesiones cariosas cuando el material se ha perdido con anterioridad. Seppa y col. ${ }^{19}$, indican que las fisuras selladas con vidrio ionomérico son más resistentes a la desmineralización, incluso después de la perdida completa del material. Esto puede ser el resultado del efecto combinado de liberación de fluoruro del ionómero y del material residual que se encuentra en la parte inferior de las fisuras.

Beiruti y col. ${ }^{20}$, estudiaron 72 fosas y fisuras de molares permanentes extraídos quirúrgicamente y compararon la profundidad de penetración del ionómero de vidrio Fuji IX de alta viscosidad empleando la técnica del ART (Tratamiento Restaurador Atraumático) y el uso de un bruñidor de bolita. Los autores concluyen indicando que con la presión digital se produce una penetración $90 \%$ del material, con respecto al bruñidor que alcanza solo un $23 \%$. 
Un estudio reportado por Amaral y col. ${ }^{21}$, demostró que el ionómero de vidrio Fuji IX es capaz de hacer la superficie del esmalte de fosas y fisuras más resistente, aumentando el valor de su dureza y remineralización, que la observada al utilizar selladores a base de resina como el Delton (Dentsply). Igualmente, observaron que los niños con alta incidencia de caries dental tenían un grado más bajo de retención.

Las controversias en los estudios descritos anteriormente, de cual es mejor material para sellar las lesiones de caries dental no cavitadas en molares permanentes, los sellantes resinosos, vidrio ionomérico de alta densidad o vidrios ionomérico modificados con resina, orientaron el propósito de la investigación, la cual fue, evaluar la efectividad del sellado de lesiones de caries dental no cavitadas utilizando un vidrio ionomérico de alta densidad 3M ESPE Ketac Molar Easymix, un sellante a base de resina 3M ESPE Climpro y un vidrio ionomérico modificado con resina de nanotecnología 3M ESPE Ketac N100 en primeros molares permanentes, empleando como indicadores clínicos la retención del material y progresión de las lesiones en primeros molares permanentes.

\section{Material y métodos}

Un total de 262 primeros molares permanentes fueron seleccionados en 74 niños con una edad media 7,47 DE \pm 0.8 años, estudiantes de educación primaria de la ciudad de Maracaibo, Estado Zulia, Venezuela. Como criterios de inclusión: Cada paciente tenía al menos dos primeros molares permanentes bilaterales con lesiones oclusales de caries dental no cavitadas correspondientes a los códigos 1 (1B, 1C), 2 (2B, 2C) y 3 del Sistema Internacional para la detección y evaluación de caries dental ICDAS II Baltimore
$2005^{22}$. Se excluyeron molares con sellantes, material de restauración, cara oclusal parcialmente erupcionada; lesiones de caries código 4, 5 y 6 de ICDAS $\mathrm{II}^{22}$, enfermedad periodontal, hipoplasia de esmalte, historia de dolor, pacientes no colaboradores o con enfermedad sistémica. Los pacientes fueron incluidos si aceptaban pertenecer al estudio, su representante legal autorizaba el tratamiento, y si firmaban un informe de consentimiento luego de explicarle el propósito del estudio, de acuerdo con los principios de la declaración de Helsinki ${ }^{23}$.

\section{Diseño Experimental}

Se realizó un ensayo clínico, aleatorio, doble ciego, controlado, con diseño de boca dividida y tres tipos de tratamiento. Los pacientes fueron asignados aleatoriamente a cada grupo de tratamiento: Tratamiento 1: vidrio ionomérico 3M ESPE Ketac Molar Easymix® St Paul MN. Tratamiento 2: Sellante resinoso 3M ESPE Climpro® St Paul MN; Tratamiento 3: vidrio ionomérico modificado con resina 3M ESPE Ketac ${ }^{\circledR}$ N100 St Paul MN. En cada tratamiento, el sellador fue colocado aleatoriamente en uno de los molares del par (Grupo experimental), su contralateral no recibió sellante, solo cepillado con pasta dental (Grupo control). La distribución de los pacientes se realizó de acuerdo a dos tablas de números aleatorios generada por la Aplicación SPSS-Windows V15.0 (SPSS, Inc, Chicago IL), la primera diseñada para asignar los pacientes a los tipos de tratamiento y la segunda para determinar la ubicación del molar que recibiría el sellante (derecho o izquierdo).

\section{Tamaño Muestral}

Se planteó un diseño experimental, con tres tratamientos e igual número de pares en cada 
uno, se empleó la información disponible sobre el parámetro de la variable "retención", la cual fue estimada en $75 \%$. Se fijó una diferencia admisible del $13 \%$, un error alfa $(\alpha)$ del $5 \%$ y un error beta $(\beta)$ del $20 \%$, para una potencia de $80 \%$; resultando un tamaño muestral por tipo de tratamiento de 88 unidades experimentales. Para el cálculo del tamaño muestral se tomo el modelo propuesto en Norman G.R. y Streiner D.L. $(1996)^{24}$.

\section{Evaluación clínica basal}

Antes del recibir el tratamiento, a los pacientes se les realizó una historia médico-odontológica y cepillado dental con dentífrico fluorurado. Para el diagnóstico visual y táctil de las lesiones se utilizó una lámpara de luz halógena, un espejo bucal plano $\mathrm{N}^{\circ}$, una sonda de la O.M.S., y aire comprimido de la jeringa triple. Las unidades de observación fueron evaluadas según los criterios ICDAS II $^{22}$ por un investigador calibrado $(k=0,86)$.

\section{Procedimiento para el sellado de las lesiones}

Un odontólogo entrenado, realizó el sellado de las lesiones no cavitadas según las indicaciones del fabricante, y otro odontólogo, se encargó de la manipulación de los materiales, verificando en los molares la presencia y adaptación del sellador en todas las fosas y fisuras oclusales. A todos los pacientes se les indicó no ingerir ningún alimento una hora después de recibir el tratamiento.

Tratamiento 1: Uno de los molares seleccionados recibió el Vidrio Ionomérico 3M ESPE Ketac ${ }^{\circledR}$ Molar Easymix St Paul MN. La proporción de polvo/liquido se empleó según el fabricante (1:1); bajo aislamiento relativo con rollos de algodón, la superficie fue secada con torundas de algodón y acondicionada con acido poliacrílico durante 10 segundos, seguido del lavado con agua y secado de la superficie. La técnica de presión digital fue utilizada para condensar el material dentro de las fosas y fisuras, durante 30 segundos realizando movimientos en sentido mesio-distal y vestibulo-lingual y/o palatino, se removieron los excesos del material con una cucharita de dentina. Después de un endurecimiento inicial la oclusión fue chequeada con papel articular (Prehma, Medeco Miami, FL) y si fuera necesario, ajustada con un excavador. Una capa de vaselina Cero (Medellín, Colombia), fue colocada sobre el material de sellado para prevenir la deshidratación.

Tratamiento 2: Uno de los molares seleccionados se les aplicó sellante de resina 3M ESPE Climpro® St Paul MN. Bajo aislamiento absoluto con grapas Hu-Friedy RDCM7, RDCM14A (Hu-Friedy Chicago, IL USA) y diques de goma ROEKO Flexi Dam Non Latex (Coltene/ Whaledent, Largenau Germany) se realizó profilaxis con cepillo dental y piedra pómez (EZ-PUMICE, Products Fesenko, Venezuela), lavado y secado de la superficie con aire de la jeringa triple, luego se realizó durante 15 segundos la técnica de grabado con ácido fosfórico al 35\% (Scotchbond® ${ }^{\circledR}$ 3M ESPE St Paul MN), se lavó por 30 segundos, se removieron con succión los residuos de agua y se secó nuevamente con aire de la jeringa triple, se aplicó el sellante en las fosas y fisuras, se fotocuró el material durante 40 segundos (lámpara de luz ultravioleta Litex ${ }^{\circledR} 680$ Dentamerica ${ }^{\circledR}$ de 450 a 525 nanómetros de longitud de onda), se retiró el aislamiento, la oclusión fué revizada con papel articular (Prehma, Medeco Miami, FL).

Tratamiento 3: Uno de los molares seleccionados aleatoriamente recibió Vidrio Ionomérico 
3M ESPE Ketac® N100 St Paul MN, color A3. Bajo aislamiento absoluto con grapas Hu-Friedy RDCM7, RDCM14A (Hu-Friedy Chicago, IL USA) y diques de goma ROEKO Flexi Dam Non Latex (Coltene/Whaledent, Largenau Germany), se secó la superficie con aire de la jeringa triple, se removieron los residuos de agua con succión, con un micro aplicador desechable (Cavibrush, extra-fine, Dentscare LTDA, Brasil) se colocó el primer acondicionador (3M ESPE Ketac ${ }^{\circledR}$ N100 Nano-Ionomer Primer) en las fosas y fisuras durante 15 segundos, se aireó con jeringa triple durante 10 segundos, se fotocuró durante 10 segundos (lámpara de luz ultravioleta Litex® 680 Dentamerica ${ }^{\circledR}$, de 450 a 525 nanómetros de longitud de onda), se dosificó el material según el fabricante, se mezcló por 20 segundos con espátula plástica (hasta lograr un color uniforme), el material se aplicó con 3M ESPE Puntas Intraorales Dispensadoras ${ }^{\circledR}$, y se compactó con un bruñidor de bolita a nivel de fosas y fisuras, se fotocuró durante 20 segundos, se retiró el aislamiento absoluto y la oclusión fue chequeada con papel articular (Prehma, Medeco Miami, FL).

\section{Evaluación de la efectividad del tratamiento}

Para evaluar la efectividad del tratamiento, ddespués de seis meses, se determinó la retención del material y el avance en severidad de las lesiones de caries dental, existentes en el estudio basal, realizada por el mismo examinador e igualdad de condiciones. Se registró la retención del sellante de acuerdo a los criterios descritos por García-Godoy ${ }^{25}$ (Tabla 1), considerando solo dos situaciones: tratamiento exitoso (sellante con retención total) y tratamiento defectuoso o fracaso (sellante con retención parcial y/o perdida completa). Las fisuras no cubiertas de los molares controles y de sellantes perdidos fueron
Tabla 1. Criterios empleados para la retención de los sellantes, según García-Godoy ${ }^{25}$.

\begin{tabular}{|c|}
\hline \multicolumn{1}{|c|}{ Retención del Sellante } \\
\hline $\begin{array}{c}\text { Retención Total: el sellante cubre todas las fosas y } \\
\text { fisuras }\end{array}$ \\
\hline Retención Parcial: el sellante cubre parcialmente \\
las fosas y fisuras \\
\hline $\begin{array}{c}\text { Perdido: pérdida completa del sellante en todas } \\
\text { las áreas de la superficie }\end{array}$ \\
\hline
\end{tabular}

examinadas para la presencia de caries según los criterios de ICDAS II Baltimore 2005 ${ }^{22}$.

\section{Análisis estadístico}

El procesamiento estadístico se realizó con la aplicación SPSS-Windows V15.0 (SPSS, Inc, Chicago IL). Se realizaron las pruebas estadísticas: Bondad de Ajuste con la distribución de Chi Cuadrado y Prueba de Kolmogorov y Smirnof (para comparar la retención por tipo de tratamiento); Test de Fisher (para contrastar la incidencia de lesiones de caries dental entre los grupos de unidades experimentales y controles); y Prueba de Independencia con la distribución de Chi cuadrado (para contrastar la incidencia de lesiones entre los tipos de tratamiento).

\section{Resultados}

De los 262 molares incluidos en el estudio, 91 molares $(34,6 \%)$ en 26 niños no lograron ser evaluados a los 6 meses; 44 molares $(16,7 \%)$ de 12 niños que fueron retirados o cambiados de plantel; 36 molares $(13,7 \%)$ de 9 niños con inasistencia los días de la evaluación; 11 molares $(4,2 \%)$ de 5 niños que recibieron otros tratamientos antes de esta evaluación. 
En la evaluación de retención de los sellantes según el tipo de tratamiento (Tabla 2) el mayor porcentaje de retención total fue de un 51,7\% para el tratamiento 1 Ketac Molar®, seguido del $43,4 \%$ para el Climpro® y $26,4 \%$ para el Ketac ${ }^{\circledR}$ N100. El sellante de ketac Molar ${ }^{\circledR}$ fue el que mostró menor tasa de perdida completa de el material seguido del Climpro®. No obstante estas diferencias no fueron estadísticamente significativas.

En cuanto a la incidencia de caries en los molares con pérdida completa del material, después de 6 meses, diez de las 14 lesiones con pérdida total del material no progresaron a un grado de mayor severidad y solo cuatro $(28,5 \%)$ avanzaron. De igual forma la incidencia de caries en los molares que sirvieron como control fue de $31,8 \%$ (Tabla 3). Después de 6 meses, la incidencia de lesiones cariosas en los molares que presentan pérdida completa del material, no difiere significativamente de la incidencia de caries en los molares que no reciben el tratamiento $(\mathrm{p}>0,05 \%)$.

De los 14 casos de los molares tratados con los tres tipos de materiales, la incidencia de lesio-

Tabla 2. Retención de los sellantes según tipo de tratamiento.

\begin{tabular}{|c|c|c|c|c|c|c|c|c|c|c|}
\hline \multirow{3}{*}{$\begin{array}{c}\text { Tipo de } \\
\text { tratamiento }\end{array}$} & \multirow{2}{*}{\multicolumn{2}{|c|}{$\begin{array}{c}\text { Tratamiento } \\
\text { exitoso }\end{array}$}} & \multicolumn{6}{|c|}{ Tratamiento defectuoso } & \multirow{2}{*}{\multicolumn{2}{|c|}{ Total }} \\
\hline & & & \multicolumn{2}{|c|}{ Parcial } & \multicolumn{2}{|c|}{ Perdido } & \multicolumn{2}{|c|}{ Parcial+Perdido } & & \\
\hline & $\mathbf{n}^{*}$ & $\%$ & $\mathbf{n}$ & $\%$ & $\mathbf{n}$ & $\%$ & $\mathbf{n}^{* *}$ & $\%$ & $\mathbf{n}$ & $\%$ \\
\hline $\begin{array}{c}\text { Ketac } \\
\text { Molar® }\end{array}$ & 15 & 51,7 & 12 & 41,4 & 2 & 6,9 & 14 & 48,3 & 29 & 100 \\
\hline Clinpro® & 10 & 43,4 & 10 & 43,5 & 3 & 13,1 & 13 & 56,6 & 23 & 100 \\
\hline Ketac ${ }^{\circledR}$ N100 & 9 & 26,4 & 16 & 47,1 & 9 & 26,4 & 25 & 73,5 & 34 & 100 \\
\hline Total & 34 & 39,5 & 38 & 44,2 & 14 & 16,3 & 52 & 60,5 & 86 & 100 \\
\hline
\end{tabular}

Prueba de Bondad de Ajuste con distribución de Chi Cuadrado entre los tratamientos, verificado con la Prueba de Kolmogorov y Smirnof.

*Tratamiento exitoso $p=0,36$.

**Tratamiento defectuoso $p=0,08$.

Tabla 3. Incidencia de caries dental entre los grupos de unidades control y experimentales.

\begin{tabular}{|c|c|c|c|c|c|c|}
\hline & \multicolumn{2}{|c|}{ Experimental } & \multicolumn{2}{c|}{ Control } & \multicolumn{3}{c|}{ Total } \\
\hline & $\mathbf{n}$ & $\%$ & $\mathbf{n}$ & $\%$ & $\mathbf{n}$ & $\%$ \\
\hline Avance de lesiones & 4 & 28,5 & 27 & 31,8 & 31 & 31,3 \\
\hline Sin avance de lesiones & 10 & 71,5 & 58 & 68,2 & 68 & 68,7 \\
\hline Total & 14 & 100 & 85 & 100 & 99 & 100 \\
\hline
\end{tabular}

Fisher $p=0,5791$

( $p>0,05 \%)$. 
Tabla 4. Proporción de la Incidencia de caries dental en los molares controles según tipo de tratamiento.

\begin{tabular}{|c|c|c|c|c|}
\hline & $\begin{array}{c}\text { Ketac Molar } \\
\text { Easymix }\end{array}$ & Clinpro ${ }^{\circledR}$ & Ketac ${ }^{\circledR}$ N100 & Total \\
\hline Avance de lesiones & $\mathbf{n}$ & $\mathbf{n}$ & $\mathbf{n}$ & $\mathbf{n}$ \\
\hline No avanzaron & 11 & 6 & 10 & 27 \\
\hline Total & 18 & 17 & 23 & 58 \\
\hline
\end{tabular}

Prueba de independencia con la distribución de Chi cuadrado. $p=0,644$

nes de caries estuvo distribuida de la siguiente manera: molares tratados con Ketac Molar ${ }^{\circledR}$, en uno de dos molares con pérdida completa del sellante la lesión avanzó a un grado de mayor severidad (de código $1 \mathrm{~b}$ a código $2 \mathrm{c}$ ). Mientras que en el tratamiento 2 Climpro®, de tres lesiones con pérdida completa del sellante, solo una progresó (de código 2c a código 3). Para el tratamiento 3 Ketac N100®, de 9 lesiones con pérdida completa del material solo 2 avanzaron (de código 1 b a 2 b y de código 2 c a 3). Las evidencias resultantes no fueron significativas.

Por otra parte, en los molares controles que no recibieron ningún tipo de material sellador, de un total de 85 lesiones se diagnosticaron 27 nuevas $(31,8 \%)$. Para los molares controles del tratamiento 1 Ketac Molar ${ }^{\circledR}$, de 29 lesiones 11 progresaron a códigos 2 (38\%), 5 a código $2 \mathrm{~b}$ (de $1 b$ a $2 b$ ), 6 a 2c (dos de $1 c$ a 2 c y cuatro de $2 b$ a 2c). En los controles para el tratamiento 2 Climproß, de 23 lesiones 6 progresaron $(26,1 \%)$, una a código $2 \mathrm{~b}$ (de $1 \mathrm{~b}$ a 2b), 4 a 2c (una de 1c a 2c y tres de $2 \mathrm{~b}$ a $2 \mathrm{c}$ ), y una a código 3 (de código $2 \mathrm{c} \mathrm{a}$ 3). Los molares controles del tratamiento $3 \mathrm{Ke}-$ tac ${ }^{\circledR} \mathrm{N} 100$, de 33 lesiones 10 avanzaron $(30,3 \%)$, 2 a código $2 \mathrm{~b}$ (de $1 \mathrm{~b}$ a $2 \mathrm{~b}$ ), 7 a código $2 \mathrm{c}$ (de $1 \mathrm{c}$ a 2c) y una a código 3 (de 2c a 3). Los resultados encontrados, en la proporción de incidencia de lesiones de caries en los molares controles de los tres tratamientos, no presentaron diferencias significativas (Tabla 4).

\section{Discusión}

La efectividad de los sellantes en la prevención de caries en la superficie oclusal de molares permanentes ha sido documentada ${ }^{25,26,27} \mathrm{y}$ aceptada en la práctica clínica ${ }^{28,29}$. Sin embargo, cuando en la superficie oclusal una lesión de mancha blanca o café puede ser detectada; la decisión de sellar la lesión sin ningún tipo de procedimiento operatorio; parece no ser una decisión fácil de adoptar por algunos profesionales. El tratamiento de elección serian las llamadas cavidades profilácticas; ocasionalmente indicarían terapia con fluoruros; pero muy pocos el sellado de la lesión sin ningún tipo de remoción.

En este estudio, tres tipos de materiales fueron evaluados para medir la efectividad del sellado de lesiones no cavitadas en la superficie oclusal de primeros molares permanentes en niños de 6 a 9 años de edad. Un sellante a base de resina de cuarta generación; y dos tipos de vidrios ionomérico, uno convencional de alta densidad; y otro modificado con resina de nanotecnología. 
Los estudios ${ }^{30,31}$ que comparan sellantes a base de resina y sellantes de vidrio ionomérico generalmente utilizan diseños de boca dividida o diseños de grupos paralelos. En este estudio se utilizaron simultáneamente el diseño de grupos paralelos, donde cada paciente recibió un tipo de material; y el diseño de boca divida, donde en cada paciente, un molar recibió tratamiento constituyendo el grupo experimental y el contralateral no recibió tratamiento sirviendo como control. Esto bajo la premisa de que los materiales utilizados; podrían incrementar la concentración del fluoruro en saliva. Es conocido que la liberación prolongada de fluoruros puede ser efectiva como medida en la prevención de caries y podría enmascarar alguna diferencia entre los materiales en una misma boca ${ }^{13,15}$.

Los estudios ${ }^{27,28,30,32}$ que comparan los sellantes a base de resina, señalan una tasa de retención completa superior a la observada en los molares sellados con vidrio ionomérico. En contraste, nuestros resultados señalan que la mayor tasa de retención completa del material se observó en los molares sellados con vidrio ionomérico convencional de alta densidad; seguido del sellante a base de resina. El material con la menor proporción de retención completa fue observado en el vidrio ionomérico modificado con resina de nanotecnología.

De los tres materiales utilizados en este estudio, el que obtuvo mayor tasa de retención completa, luego de 6 meses, fue el Ketac Molar Easymix ${ }^{\circledR}$ $(51,7 \%)$, un vidrio de alta densidad, cuyas propiedades físicas han sido mejoradas, resistencia a la compresión, y resistencia a la flexión, resistencia al desgaste; adhesión al esmalte y dentina, liberación de fluoruro y resistencia a la erosión por ácidos. Está indicado como terapia de obturación de mínima intervención, tratamiento de restauración atraumático y como sellante de fosas y fisuras. El otro material fue el Climpro® (43,4\% de retención total); material sellador a base de resina constituye una resina fluida; de cuarta generación con capacidad de liberar fluoruro; esto hace que el material tenga menor resistencia a la compresión y mayor capacidad de desgaste por la liberación de fluoruro. El tercer material fue el Ketac ${ }^{\circledR}$ N100 (26,4\% de retención total); en una presentación pasta-pasta, constituye un vidrio ionomérico fotopolimerizable, modificado con resina de nano-relleno, esta combinación permite mejorar la estética y a la vez constituye un material que permite la liberación de fluoruro ${ }^{33}$.

La literatura ${ }^{26,34,35}$ reporta diversos factores que pueden influir en la tasa de retención de los materiales. Para controlar estos factores; en este estudio se seleccionaron únicamente primeros molares permanentes. La contaminación accidental por la saliva, es un factor crítico para algunos materiales, en nuestro estudio; los sellantes a base de resina y de ionómeros modificados con resina fueron colocados bajo aislamiento absoluto mientras; que el sellante de vidrio ionomérico bajo aislamiento relativo con rollos de algodón; siguiendo el procedimiento descrito para la colocación de sellantes con la técnica de tratamiento restaurador atraumático ${ }^{16,36}$.

En este sentido, un estudio comparativo ${ }^{37}$ sobre la aplicación de sellantes resinosos bajo aislamiento absoluto y bajo aislamiento relativo, demostró que no existen diferencias significativas en las tasas de retención y no recomienda el uso de aislamiento absoluto solo para la aplicación de un sellante debido a la frecuente necesidad de colocar anestesia local, sumado a la incomodidad del paciente por el uso de las grapas. De acuerdo; con esta evidencia, una revisión sistemática publicada en el 2006 por Muller-Bolla y col. ${ }^{38}$, sobre la retención de los sellantes resino- 
sos, indica que el uso del aislamiento absoluto no afecta en la retención de este tipo de sellantes.

Otro aspecto importante a considerar en la evaluación de un material sellador, es su capacidad de prevenir o detener el desarrollo de una lesión de caries dental; y el efecto que pueda dejar un material después de haberse perdido. En virtud de esto, Ashwin y Arathi ${ }^{39}$ indican que el uso de los sellantes permite la liberación fluoruros lo que incrementa la resistencia a la desmineralización, disminuyendo así, el desarrollo de lesiones de caries.

En este estudio, después de 6 meses, de las 14 lesiones de caries dental detectadas en los molares con perdida completa del sellante; solo 4 lesiones de caries avanzaron a un grado de mayor severidad con una proporción de $28,5 \%$ comparada con un $31,8 \%$ de lesiones que avanzaron en los molares que no recibieron tratamiento; estas diferencias no fueron estadísticamente significativas. Sin embargo, Oong y col. ${ }^{11}$; reportaron que al colocar un sellante sobre una lesión cariosa, las bacterias existentes en las fosas y fisuras de los molares son aisladas del medio y de los fluidos bucales, las bacterias no parecen ser capaces de ejercer su potencial cariogénico, debido a que no tienen acceso a los sustratos fermentables, lo cual conlleva a una reducción de estos organismos, mostrando una disminución en el progreso de la lesión.

De acuerdo con esto; podríamos inferir que en los molares con retención completa y parcial del material las lesiones se mantienen sin avance, considerándose que de 86 molares que fueron sellados solo 4 lesiones de caries progresaron; $4,7 \%$ de avance de las lesiones que reciben tratamiento; comparado al 31,8\% de los molares que no reciben tratamiento.

Nuestros resultados también contrastan con lo reportado por Griffin y col. ${ }^{12}$ un meta-análisis sobre la efectividad de los sellantes en el manejo de lesiones de caries, ellos reportan un porcentaje de progresión anual de las lesiones selladas de un $2,6 \%$ y un $12,6 \%$ para los dientes que no son sellados; los valores son mayores en nuestro estudio; esta discrepancia podría explicarse por la diferencia de criterios utilizados para el diagnostico de caries dental. Los criterios de diagnóstico de caries dental utilizados en el estudio fue el ICDAS II ${ }^{22}$; un sistema que permite detectar cambios menores en las lesiones.

\section{Conclusiones}

El vidrio ionomérico Ketac Molar Easymix®® de alta densidad, mostró una mayor tasa de retención que el sellante a base de resina Climproß, y el vidrio ionomérico modificado con resina de nanotecnología Ketac ${ }^{\circledR}$ N100.

No se observaron evidencias de diferencias estadísticamente significativas en la retención, después de seis meses, entre los tipos de tratamiento, Ketac Molar Easymix® $(51,7 \%)$ seguido del Climpro $(43,3 \%)$ y del Ketac® N100 $(26,4 \%)$.

La incidencia de lesiones de caries dental, en los molares donde se perdió completamente el sellante fue de un $28,5 \%$ frente a un $31,8 \%$ de los molares controles que no recibieron el sellante. 


\section{Referencias}

1. Beauchamp J., Caufield P., Crall J., Donly K., Feigal R., Gooch B., Ismail A.,

2. Kohn W., Siegal M., Simonsen R. Evidence-Based Clinical recommendations for the Use of Pit-and-Fissure Sealants: A Report of the American Dental Association Council on Scientific Affairs. J Am Dent Assoc 2008;139;257-268.

3. Aguilar FG, Drubi-Filho B, Casemiro LA, Watanabe M, Pires-de-Souza F. Retention and penetration of a conventional resin-based sealant and a photochromatic flowable composite resin placed on occlusal pits and fissures. J Indian Soc Pedod Prev Dent 2007;25:169-73.

4. Clinical Affairs Committee - Restorative Dentistry Subcommittee. Guideline on Pediatric Restorative Dentistry. American academy of pediatric dentistry. REFERENCE MANUAL V 33 / NO 611 / 12.

5. Celiberti P, Lussi A. Penetrationability and microleakage of a fissure sealant applied on artificial and natural enamel fissure caries. Journal of Dentistry, 2007;Vol. 35, Issue 1, January,59-67.

6. Welbury R, Raadal M, Lygidakis N. EAPD guidelines for the use of pit and fissure sealants. European journal of paediatric dentistry, 2004/3, 179-184.

7. Oulis C, Berdouses E, Mamai - Homata E, Polychoronopoulou A. Prevalence of sealants in relation to dental caries on the permanent molars of 12 and 15-year-old Greek adolescents. A national Pathfinder survey. BMC Public Health 2011,11:100.

8. Kavaloglu S, Sandalli N. Compressive Strength, Surface Roughness, Fluoride Release and Recharge of FourNew Fluoride-releasing Fissure Sealants. Dental Materials Journal 2007;26(3): 335-341.

9. Ahovuo-Saloranta, Hiiri A, Nordbland A, Makela M, Worthington HV. Pit and fissure sealants for preventing dental decay in the permanent teeth of children and adolescents. Cochrane Database Syst Rev 2010,8(4):CD001830.

10. Azarpazhooh A, Main PA. Pit and fissure sealants in the prevention of dental caries in children and adolescents: a systematic review. J Can Dent Assoc. 2008 Mar;74(2):171-7.

11. Simonsen RJ. Pit and Fissure sealant: review of literature. Pediatric Dent. 2002 Sep-Oct;24(5):393-414.

12. Oong E, Griffin S, Kohn W, Gooch B, Caunfield P. The effect of dental sealants on bacteria levels in caries lesions. A review of the evidence. JADA 2008,139(3):271-278.

13. Griffin S, Oong E., Kohn W., Vidakovic B., Gooch B., and CDC Dental Sealant Systematic Review Work Group: Bader J, Clarkson J, Fontana M, Meyer D, Rozier R, Weintraub J, Zero D. The effectiveness of sealants in managing caries lesions. J Dent Res. 2008 Feb;87(2):169-74.

14. Salar DV., García-Godoy F., Flaitz CM., Hicks MJ. Potential inhibition of demineralization in vitro by fluoride-releasing sealants. J Am Dent Assoc. 2007 Apr;138(4):502-6.

15. Trairatvorakul C., Kladkaew S., Songsiripradabboon S. Active Management of Incipient Caries and Choice of Materials. J Dent Res 2008 Mar;87(3):228-232.

16. Kantovitz KR., Pascon FM., Correr GM., Borges AF., Uchoa MN., Puppin-Rontani RM. Inhibition of mineral loss at the enamel/sealant interface of fissures sealed with fluoride- and non-fluoride containing dental materials in vitro. Acta Odontol Scand. 2006 Nov;64(6):376-83.

17. Beiruti N., Frencken JE., van't Hof MA., Taifour D., van Palenstein Helderman WH. Caries-preventive effect of a onetime application of composite resin and glass ionomer sealants after 5 years. Caries Res. 2006;40(1):52-9.

18. Beiruti N., Frencken JE., van 't Hof MA., van Palenstein Helderman WH. Caries-preventive effect of resin-based and glass ionomer sealants over time: a systematic review. Community Dent Oral Epidemiol. 2006 Dec; 34(6):403-9.

19. Komatsu H., Shimokobe H., Kawakami S., Yoshimura M. Caries-preventive effect of glass ionómero sealant reapplication: Study presents three-year results. J Am Dent Assoc 1994;125(5):543-9.

20. Seppa L., Forss H. Resistance of oclusal fissures to desmineralization after loss of glass ionomer sealants in vitro. Pediatr Dent 1991;13(1):39-42.

21. Beiruti N., Frencken J., Mulder J. Comparison between two glass-ionomer sealants placed using finger pressure (ART approach) and a ball burnisher. Am J Dent. 2006 Jun;19(3):159-62. 
22. Amaral M., Guedes-Pinto A., Chevitarese O. Effects of a glass-ionomer cement on the remineralization of occlusal caries - an in situ study. Braz. oral res. 2006; vol.20 no.2 São Paulo Apr./June.

23. International Caries Detection \& Assessment System Coordinating Committee. The International Caries Detection and Assessment System (ICDAS II at June 2005). July 2005. Disponible en: www.icdas.org.

24. Declaración de Helsinki: principios éticos para la investigación médica sobre sujetos humanos. Análisis de la $5^{\mathrm{a}}$ Reforma, aprobada por la Asamblea General, de la Asociación Médica Mundial en octubre del año 2000, en Edimburgo, Respecto del texto aprobado en Somerset West (Sudáfrica) en octubre de 1996. Acta Bioética, 2000, año VI, nº 2.

25. Norman G., Streiner D. Bioestadística. Edición en Español, Mosby Doyma Libros S.A. Madrid España. 1996. Pág. 61-62

26. García-Godoy F. Retention of a light-cured fissure sealant (Helioseal) in a tropical environment after 12 months. Clin Prev Dent 1986;8:11-3

27. Pinar A., Sepet E., Aren G., Bölükbasi N., Ulukapi H., Turan N. Clinical performance of sealants with and without a bonding agent. Quintessence Int 2005;36:355-60.

28. Arrow P., Riordan P. Retention and caries preventive effects of a glass ionómer cement and a resin-based fissure sealant. Community Dent Oral Epidemiol 1995;23:282-5.

29. Hiiri A., Ahovuo-Saloranta A., NordbladA., Makela M. Pit and fissure sealants versus fluoride varnishes for preventing dental decay in children and adolescents. Cochrane Database Syst Rev. 2006 Oct 18;(4):CD003067.

30. Rethman J. Trends in preventive care:Caries risk assessment and indications for sealants. J Am Dent Assoc 2000;131(suppl):8S-12S.

31. Poulsen S., Beiruti N., Sadat N. A comparison of retention and the effect on caries of fissure sealing with a glass ionómero and a resin-based sealant. Community Dent Oral Epidemiol. 2001;29:298-301.

32. Simonsen RJ. Glass ionómer as fissure sealant: A critical review. J Public Health Dent. 1996;56:146-9.

33. Ganesh M., Shobha T. Comparative evaluation of the marginal sealing ability of Fuji VII and Concise as pit and fissure sealants. J Contemp Dent Pract. 2007 May 1;8(4):10-8.

34. Henestroza G. Adhesión en Odontología Restauradora. Asociación Latinoamericana de Operatoria Dental y Biomateriales. Editorial MAIO. Brasil. 2003

35. Waggoner W., Siegal M. Pit and fissure sealant application: Updating the technique. J Am Dent Assoc 1996;127:351-61.

36. Llodra J., Bravo M., Delgado-Rodriguez M., Baca P., Galvez R. Factors influencing the effectiveness of sealants: A meta-analysis. Community Dent Oral Epidemiol 1993;21:261-8.

37. Vieira A., Melo N., Bresciani E., Barata T., da Silva S., Machado M., de Lima M. Evaluation of glass ionomer sealants placed according to the ART approach in a community with high caries experience: 1-year follow-up. J appl Oral Sci 2006 Aug;14(4):270-5.

38. Albani F., Ballesio I., Campanella V., Marzo G. Pit and fissure sealants: Results at five and 10 years. Eur J Paediatr Dent 2005;6:61-5.

39. Muller-Bolla M, Lupi-Pégurier L, Tardieu C, Velly AM, Antomarchi C. Retention of resin-based pit and fissure sealants: A systematic review. Community Dent Oral Epidemiol. 2006 Oct;34(5):321-36.

40. Ashwin R, Arathi R. Comparative evaluation for microleakage between Fuji-VII glass ionomer cement and light-cured unfilled resin: A combined in vivo in vitro study. J Indian Soc Prev Dent. 2007 Apr-Jun;25(2):86-7

Recibido: 28-11-2013

Aceptado: 06-12-2013

Corerespondencia: Jesús Alberto Luengo Fereira.jluengofereira@gmail.com

Carretera a la Bufa S/N, Colonia Centro. Especialidad en Odontopediatría. Unidad Académica de Odontología. Universidad Autónoma de Zacatecas. Zacatecas, México. Teléfono/ fax: +52-492-9229650 